\title{
Factores de riesgo de lesión por accidentes de tráfico y el impacto de una intervención sobre la
}

\section{carretera}

Risk factors of injury by traffic accidents and the impact of an intervention on the road

Martha C Híjar-Medina, Carlos E Carrillo-Ordaz, Mario E Flores-Aldana, Rafael Anaya y Ma. Victoria López-López

Centro de Investigación en Sistemas de Salud. Instituto Nacional de Salud Pública. Cuernavaca, México

HIJAR-MEDINA Martha C, Carlos E Carrillo-Ordaz, Mario E Flores-Aldana, Rafael Anaya y Ma. Victoria LópezLópez Factores de riesgo de lesión por accidentes de tráfico y el impacto de una intervención sobre la carretera Rev. Saúde Públeca, 33 (5), 1999 www.fsp.usp.br/rsp 


\title{
Factores de riesgo de lesión por accidentes de tráfico y el impacto de una intervención sobre la carretera* Risk factors of injury by traffic accidents and the impact of an intervention on the road
}

\author{
Martha C Híjar-Medina, Carlos E Carrillo-0 rdaz, Mario E Flores-Aldana, Rafael \\ Anaya y Ma. Victoria López-López
}

Centro de Investigación en Sistemas de Salud. Instituto Nacional de Salud Pública. Cuernavaca, M éxico

\section{Descriptores}

Accidentes de transito.

Heridas y traumatismos, epidemiología.

Carreteras, normas.

\section{Keywords}

Accidents, traffic.

Wounds and injuries, epidemiology. Roads standards.

\section{Resumen}

\section{Objetivo}

Evaluar el efecto de intervenciones realizadas en la autopista, tanto en la ocurrencia de lesiones por accidentes de tráfico, como en la gravedad de las mismas.

\section{Métodos}

Se realizó un análisis comparativo de dos estudios transversales realizados en 1994 y 1996 en los que se investigó la ocurrencia de lesiones en los conductores de vehículos a motor que sufrieron accidente en la autopista, Cuernavaca, México.

\section{Resultados}

Para 1994 la tasa de accidentes fue de 7,96 / 100.000 vehículos y para 1996 fue de 8,49 / 100.000 vehículos, incremento no significativo (p>0,05). Para 1994 la tasa de lesionados fue de 2,10 / 100.000 vehículos y para 1996 de 1,35 / 100.000 vehículos, disminución significativa ( $\mathrm{p}<0,000)$. El autorreporte de uso del cinturón $(63,46$ vs $76,6 \%)$, los vehículos pequeños accidentados (7,9 vs 37,7\%), horario nocturno (23,7 vs 31,8\%) y el sentido México-Cuernavaca (45 vs 66,7\%), fueron mayores en 1996 ( $\mathrm{p}<0,05)$. El riesgo de lesión entre los expuestos a las intervenciones en la autopista (1996) y los no expuestos (1994), ajustado por edad, uso de cinturón, velocidad, ingestión de alcohol y causa externa, empleando un modelo de regresión logística, presentó un efecto protector (RM 0,42 IC95\% 0,27 - 0,66).

\section{Conclusión}

Es evidente la necesidad de abordajes multisectoriales en el estudio y en la evaluación de las intervenciones en el campo de los accidentes de tráfico, siendo los resultados de la investigación un ejemplo claro de las repercusiones en salud que pueden tener las intervenciones del sector comunicaciones en una autopista.

\author{
Abstract \\ Objective \\ To evaluate the effect of interventions at a highway, in the occurrence and severity of \\ injuries by traffic accidents. \\ Method \\ It was made a comparative analysis of two cross-sectional studies in 1994 and 1996.
}

Correspondencia para /Correspondence to: Martha C Híjar-Medina

Av. Universidad 655, Col. Sta. María Ahuacatitlán, Cuernavaca, Mor. México CP 62508
*Proyecto parcialmente financiado por: Organización Panamericana de la Salud. HDP/ HDR/HPP/RG/M EX/1042, y por el Southern California Injury Prevention Research Center, University of California at Los Angeles.

Recibido en 3.8.1998. Reapresentado en 19.1.1999. Aprobado en 15.4.1999. 


\section{Results}

In 1994 the rate was 7.96 accidents/ 100,000 vehicles and in 19968.49 / 100,000 vehicles. The increase was not significant $(p>0.05)$. The rate of injured drivers in 1994 was of 2.10/100,000 vehicles and of 1.35 / 100,000 vehicles in 1996, which was a significant decrease $(p<0.000)$. The self-report of use of seat belt $(63.46 \%$ versus $76.6 \%)$, the small vehicles involved in accidents (7.9\% versus $37.7 \%)$, nocturnal schedule (23.7\% versus $31.8 \%$ ) and in Mexico-Cuernavaca direction (45\% versus $66.7 \%)$, were more frequent in 1996 ( $p<0.05)$. The risk of injury, using a logistic regression model, between drivers exposed to the interventions (1996) and those that were not exposed (1994) adjusted by: age, speed, use of seat belt, alcohol intake and external cause, showed a protective effect of the interventions at the highway (OR 0.42 C195\% 0.27-0.66).

Conclusion

There is an evident need of multisectorial approaches in the study and evaluation of the interventions in the field of the traffic accidents. The present research is a clear example of the repercussions over health of interventions developed by the transportation sector at the highway.

\section{Descritores}

Acidentes de trânsito. Ferimentos e lesões, epidemiologia. Estradas, normas.

\section{Resumo}

\section{Objetivo}

Avaliar o efeito das intervenções em uma rodovia, na ocorrência e gravidade de ferimentos por acidente.

Métodos

Foi feita uma análise comparativa de dois estudos transversais em 1994 e 1996.

\section{Resultados}

Em 1994 a taxa foi de 7,96 acidentes/100.000 veículos e em 1996, 8,49/100.000 veículos. O aumento não foi significante ( $\mathrm{p}>0.05$ ). O índice de motoristas feridos em 1994 foi de 2,10/100.000 veículos e de 1,35/100.000 veículos em 1996, o que representa uma redução significante $(\mathrm{p}<0.000)$. A notificação espontânea do uso de cinto de segurança $(63,46 \%$ contra $76,6 \%)$, o número de veículos de pequeno porte acidentados $(7,9 \%$ contra $37,7 \%)$, o trânsito em horário noturno $(23,7 \%$ contra $31,8 \%)$ e a direção no sentido México-Cuernevaca (45\% contra 66,7\%) foram maiores em 1996 ( $p<0.05)$. O risco de ferimento - usando um modelo de regressão logística - entre motoristas expostos a intervenções (1996) e motoristas não expostos (1994) ajustados por: idade, velocidade, uso do cinto de segurança, ingestão de álcool e causas externas mostrou um efeito protetor das intervenções na rodovia.

\section{Conclusão}

Há uma evidente necessidade de abordagens multisetoriais no estudo e na avaliação das intervenções no campo dos acidentes de trânsito, sendo o presente estudo um exemplo claro das repercussões sobre a saúde geradas pelas intervenções desenvolvidas pelo setor de transportes na rodovia.

\section{INTRO DUCCIÓ N}

En la medida que los países se modernizan y mejoran su nivel de desarrollo, la tasa de motorización sigue un patrón directamente proporcional a este fenómeno, hecho que ha impactado, entre otros, en el ámbito de la seguridad vial ${ }^{2}$, convirtiendo a las lesiones por accidentes de tráfico en un problema de Salud Pública reconocido a nivel mundial ${ }^{1,11,14,16}$.

Las consecuencias de este tipo de accidentes, medidas en muertes, demanda de atención de urgencia prehospitalaria y hospitalaria, necesidades de recursos especializados para la atención médica y de rehabilitación, así como en las secuelas, la discapacidad generada, los años de vida potencial perdidos $^{5}$, y finalmente en su impacto en la familia y la sociedad en general, al afectar fundamentalmente la calidad de vida. Esto ha llevado a que algunos organismos internacionales reconozcan el costo social y económico que representa y a la sobrecarga que producen en los ya saturados sistemas de salud.

Este hecho justifica ampliamente el desarrollo de proyectos dirigidos a analizar sus causas, identificando los factores de riesgo específicos ${ }^{1,6,11,14,16}$ en cada uno de los ámbitos donde éstos ocurren, con el fin de plantear medidas de prevención factibles y concretas $^{7,13}$. En el caso concreto de los que ocurren 
en carretera, los factores que intervienen en la ocurrencia de lesiones, su prevención y atención son diferentes a los que ocurren en un área urbana, tanto en términos de letalidad, como en posibilidades de atención oportuna ${ }^{6,11}$.

Para el estudio de dichos factores de riesgo, durante 1994 se realizó una investigación en la autopista México-Cuernavaca con base en un diseño transversal que tuvo como objetivo el conocer la asociación entre las características del conductor, del vehículo y del medio ambiente físico con la gravedad de las lesiones traumáticas provocadas por accidentes de tráfico de vehículos a motor ${ }^{8-10}$.

Al termino de la investigación y durante el período comprendido entre finales de 1994 y principios de 1996, Caminos y Puentes Federales de Ingresos y Servicios Conexos (CAPUFE, organismo gubernamental encargado de la operación y mantenimiento de la autopista) realizó una serie de intervenciones en la autopista estudiada. Estas intervenciones se ubican dentro de la prevención primaria: señalizaciones luminosas, semáforos preventivos y vibradores en el asfalto; y de prevención secundaria: sustitución de barras metálicas laterales por muro lateral con diseño ergonómico similar al muro central.

Con el objeto de evaluar si dichas intervenciones tuvieron un efecto sobre la ocurrencia de lesiones por accidentes de tráfico, se realizó una nueva investigación en la autopista durante el período marzo a octubre de 1996.

\section{MÉTODOS}

La investigación consistió en un análisis comparativo entre dos estudios transversales realizados entre los meses de marzo y septiembre de 1994 y 1996 en los que se investigó la ocurrencia de lesiones en los conductores de vehículos a motor que sufrieron un accidente en la autopista México-Cuernavaca. Esta autopista es una de las más importantes vías de acceso a la Cd. de México, ya que representa la unión del centro del país con la zona sur hacia el Pacífico. Se desarrolla a través de 61 km por una porción del Eje Neovolcánico Transversal, con alturas que van desde los $2.250 \mathrm{~m}$ sobre el nivel del mar (m.s.n.m.) en el kilómetro 19 y 1.750 m.s.n.m. en el km 80, con el punto más alto en 3.000 m.s.n.m. en el kilómetro 46. Estas diferencias de altitud dan lugar a rectas y curvas además de pendientes muy pronunciadas.

Las investigaciones tuvieron como unidad de observación y análisis a los conductores de vehículos a motor que hubiesen sufrido un accidente en la autopista México-Cuernavaca, centrándose en los casos que recibieron atención prehospitalaria por los servicios médicos proporcionados por ambulancias de CAPUFE. Encuestadores previamente capacitados viajaban en las ambulancias de CAPUFE en el momento en que se prestaba atención prehospitalaria, y obtenían informaciónes sobre características del accidente, condiciones ambientales, y características del vehículo(s) involucrado(s). La recolección de la información se realizó mediante un cuestionario diseñado específicamente, el mismo que fue utilizado en ambas investigaciones. La información se obtuvo en el sitio del accidente por medio de un interrogatorio al conductor así como la realización de una exploración física para identificar la presencia de lesiones. Cuando no fue posible obtener la información directamente del conductor se entrevistó a un acompañante o testigo. La información relacionada con los individuos hospitalizados que no pudo obtenerse en el sitio, se recabó posteriormente en la unidad hospitalaria o en el servicio médico forense.

Los grupos de comparación se definieron en relación con la presencia de lesiones (variable dependiente), tomando como grupo de referencia a los conductores de vehículos que no presentaron lesiones como consecuencia de un hecho accidental, categorizándoles como: 1) Lesionados cuando el conductor presentaba lesiones que fueron atendidas en el sitio del accidente o que requirieron su traslado a una unidad hospitalaria para su atención, o bien fallecieron y 2) No lesionados, cuando a la exploración física no se detectaron lesiones físicas.

Las variables que fueron comunes a ambos estudios y a partir de las cuales se realizó el análisis, objeto del presente trabajo fueron:

a) Del conductor: edad (años), sexo, años de experiencia como conductor, tiempo continuo de manejo hasta la ocurrencia del accidente (horas), uso de cinturón de seguridad, velocidad a la que transitaba al momento del accidente $(\mathrm{km} / \mathrm{hr})$, consumo de alcohol en las anteriores $6 \mathrm{hrs}$. medida por autorreporte.

b) Del vehículo: modelo (año de fabricación) y marca, tamaño - determinado por la distancia entre ejes especificado por el fabricante - categorizado en chicos: $240-250 \mathrm{~cm}$, medianos: 251-290 cm, grandes: $291-340 \mathrm{~cm}$, y camiones: $>340 \mathrm{~cm}$, así como los que contaron con cinturón de seguridad.

c) Del medio ambiente: hora, día de la semana y mes del accidente, kilómetro (con una precisión de cien metros), sentido de circulación (México-Cuernavaca o CuernavacaMéxico), condiciones climatológicas al momento del accidente (normales o adversas: pavimento mojado, lluvia, niebla etc., o alguna combinación de ellas). De acuerdo a sus características físicas la carretera se categorizó en los siguientes segmentos: recta ascendente; recta descendente; curva izquierda ascendente; curva izquierda descendente; curva derecha ascendente; y curva derecha descendente.

Finalmente se obtuvo una descripción de la forma en que ocurrió el accidente y se codificó como causa externa de acuerdo a la Clasificación Internacional de Enfermedades IX Revisión (CIE IX Rev.): en E812 Colisión entre vehículos, E815 Choque con objeto fijo, E816 Salida del camino.

\section{Análisis Estadística}

Con base en la información sobre el aforo de vehículos que transitaron por la autopista durante 1994 y 1996 en los períodos estudiados, se calcularon las tasas de accidentes y 
las tasas de lesionados por 100.000 vehículos que transitaron, realizando posteriormente una comparación entre ambas, estimando las diferencias de tasas, tanto global como por tramo o sentido de la autopista.

Se realizó un estudio descriptivo de las variables continuas por medio de medidas de tendencia central y para las variables categóricas por frecuencias relativas. La comparación de tasas de accidentes y de lesionados se realizó mediante $\mathbf{t}$ de student. Se realizaron análisis de diferencia de medias por $\mathbf{t}$ de student y de proporciones por Chías ${ }^{2}$ entre ambos períodos de estudio en forma global y categorizada por sentido de circulación. En cada uno de los períodos se estimó el riesgo de lesiones para cada variable por medio del cálculo de las razones de momios (RM). Se consideró significativa una $\mathrm{p}<0,05$ y un intervalo de confianza del $95 \%$.

Finalmente, con el fin de evaluar el efecto de las modificaciones a la carretera que se realizaron entre los dos períodos, se elaboró un análisis comparativo entre ambos años de los riesgos de lesión por medio de un análisis multivariado de regresión logística considerando como expuestos a la intervención a los conductores que circularon por la autopista durante 1996 utilizando las variables que fueron significativas en el análisis bivariado como modelo saturado, siguiendo un procedimiento tipo "backward". Para el procesamiento de la información se utilizó Stata versión 5.0 (30).

\section{RESU LTAD O S}

Entre marzo y septiembre de 1994 se registraron 324 eventos provocados por accidentes de tráfico en la autopista México-Cuernavaca correspondientes a 380 conductores, con una tasa de 7,69 accidentes por 100.000 vehículos.

Para el año de 1996 el número de eventos accidentales registrados fue de 362 , correspondientes a 396 conductores, donde la tasa de accidentes fue de 8,49 / 100.000 vehículos, incremento no significativo en relación al anterior año estudiado $(\mathrm{p}>0,10)$.

En relación con el riesgo de sufrir una lesión como consecuencia del accidente, se obtuvo lo siguiente: en 1994 resultaron lesionados 104 conductores (27,37\%), con una tasa de lesionados de 2,10/ 100.000 vehículos, mientras que para 1996 fue de 63 conductores lesionados $(15,91 \%)$ y una tasa de 1,35 / 100.000 vehículos. Lo anterior representó una disminución significativa en relación al año anterior, $(\mathrm{p}<0,000)$ (Tabla 1).

Las diferencias que se encontraron en 1996 en relación al año anterior fueron: una mayor proporción en el autorreporte del uso del cinturón (63,46 vs $76,6 \%$ ), una mayor proporción en los vehículos pequeños accidentados (7,9 vs $37,7 \%)$, mayor
Tabla 1 - Tasas de accidentes y lesiones autopista MéxicoCuernavaca.1994-1996.

\begin{tabular}{|c|c|c|c|}
\hline Tasas & 1994 & 1996 & $p$ \\
\hline $\begin{array}{l}\text { Total de conductores } \\
\text { de automóviles }\end{array}$ & 380 & 396 & \\
\hline $\begin{array}{l}\text { Total de conductores } \\
\text { lesionados }\end{array}$ & $\begin{array}{r}104 \\
27,4 \%\end{array}$ & $\begin{array}{r}63 \\
15,9 \%\end{array}$ & $0,000 *$ \\
\hline $\begin{array}{l}\text { Total de conductores } \\
\text { muertos } \\
\text { Tasa de accidentes }\end{array}$ & $\begin{array}{r}6 \\
2,11 \%\end{array}$ & $\begin{array}{r}8 \\
2,02 \%\end{array}$ & 0,934 \\
\hline $\begin{array}{l}\text { x } 100.000 \text { vehículos } \\
\text { global } \\
\text { M éxico-Cuernavaca } \\
\text { Cuernavaca-M éxico }\end{array}$ & $\begin{array}{l}7,69 \\
6,92 \\
8,46\end{array}$ & $\begin{array}{r}8,49 \\
11,32 \\
5,66\end{array}$ & $\begin{array}{l}0,1674 \\
0,0000^{*} \\
0,0003^{*}\end{array}$ \\
\hline $\begin{array}{l}\text { Tasa de lesionados } \\
\text { x } 100.000 \text { vehículos } \\
\text { global } \\
\text { M éxico-Cuernavaca } \\
\text { Cuernavaca-M éxico }\end{array}$ & $\begin{array}{l}2,10 \\
1,86 \\
2,34\end{array}$ & $\begin{array}{l}1,35 \\
1,59 \\
0,77\end{array}$ & $\begin{array}{l}0,0050^{*} \\
0,4686 \\
0,0000^{*}\end{array}$ \\
\hline
\end{tabular}

ocurrencia en horario nocturno ( 23,7 vs $31,8 \%$ ) y en el sentido México-Cuernavaca (45 vs $66,7 \%$ ), $(\mathrm{p}<0,05)$ (Tabla 2).

Como se observa en la Tabla 3, en ambos estudios las variables asociadas con el hecho de resultar lesionado como consecuencia de sufrir un accidente de tráfico en la autopista fueron: la edad menor de 25 años, la no utilización del cinturón de seguridad, el transitar a una velocidad superior a los $90 \mathrm{~km} / \mathrm{hr}$, el consumo de alcohol, y el choque con objeto fijo. Ninguna de las variables del vehículo y del medio ambiente estuvieron asociadas con resultar lesionado. En 1996 la ocurrencia nocturna del accidente si se encontró asociada a la presencia de lesiones. La ingestión de alcohol y la velocidad de tránsito tuvieron una variación significativa.

$\mathrm{Al}$ ajustar el riesgo de lesión entre los no expuestos a las intervenciones de la carretera (1994) y los que fueron expuestos (1996), mediante un modelo de regresión logística, se encontró que las intervenciones realizadas en la autopista tuvieron un efecto protector para los conductores expuestos (RM 0,42, IC95\% 0,27 - 0,66). El modelo permite estimar un valor probabilístico de lesión fue significativamente menor en 1996 con relación a 1994 $(0,14$ vs $0,25, \mathrm{p}<0,000)$ (Figura). Las variables: edad menor de 25 años, el no uso del cinturón de seguridad, velocidad de conducción superior a los límites permitidos, el consumo de alcohol y el choque con un objeto fijo continúan siendo factores de riesgo significativo, aunque en valor puntual menor a los obtenidos para 1994, a excepción de la velocidad de tránsito (Tabla 4 ). 
Tabla 2 - Diferencia de proporciones de las variables analizadas segun año de estudio. Autopsia M éxico - Cuernavaca, 1994-1996.

\begin{tabular}{|c|c|c|c|}
\hline Variable & $1994(\%)$ & $1996(\%)$ & p \\
\hline $\begin{array}{l}\text { Edad (años) } \\
<25 \\
25-34 \\
35-44 \\
45-54 \\
55 \text { y }+\end{array}$ & $\begin{array}{r}19,60 \\
35,51 \\
26,99 \\
11,65 \\
6,25\end{array}$ & $\begin{array}{r}17,02 \\
30,85 \\
29.26 \\
13,03 \\
9,84\end{array}$ & 0,252 \\
\hline \multicolumn{4}{|l|}{ Sexo } \\
\hline $\begin{array}{l}\text { Femenino } \\
\text { Experiencia como conductor (media años) } \\
\text { Uso del cinturón }\end{array}$ & $\begin{array}{r}9,81 \\
13,49 \\
63,46\end{array}$ & $\begin{array}{r}9,61 \\
15,39 \\
76,66\end{array}$ & $\begin{array}{l}0,016 * \\
0,000 *\end{array}$ \\
\hline $\begin{array}{l}\text { Velocidad de tránsito } \\
£ 90 \mathrm{~km} / \mathrm{hr} \\
>90 \mathrm{~km} / \mathrm{hr} \\
\text { Consumo de alcohol }\end{array}$ & $\begin{array}{r}79,94 \\
20,06 \\
8,94\end{array}$ & $\begin{array}{l}85,32 \\
14,68 \\
10,46\end{array}$ & $\begin{array}{l}0,059 \\
0,489\end{array}$ \\
\hline $\begin{array}{l}\text { Distancia ejes }(\mathbf{c m}) \\
240-250 \\
251-290 \\
291-340 \\
>340\end{array}$ & $\begin{array}{r}7,92 \\
64,38 \\
18,21 \\
9,50\end{array}$ & $\begin{array}{r}37,72 \\
33,92 \\
20,00 \\
8,35\end{array}$ & $0,000 *$ \\
\hline $\begin{array}{l}\text { Horario de ocurrencia } \\
\text { Diurno } \\
\text { Nocturno }\end{array}$ & $\begin{array}{l}76,32 \\
23,68\end{array}$ & $\begin{array}{l}68,18 \\
31,82\end{array}$ & $0,011 *$ \\
\hline $\begin{array}{l}\text { Día de la semana } \\
\text { Hábil } \\
\text { Fin de semana }\end{array}$ & $\begin{array}{l}57,37 \\
42,63\end{array}$ & $\begin{array}{l}58,59 \\
41,41\end{array}$ & 0,731 \\
\hline \multirow{2}{*}{$\begin{array}{l}\text { Condiciones ambientales adversas } \\
\text { Sentido de circulación } \\
\text { México-Cuernavaca } \\
\text { Cuernavaca-M éxico }\end{array}$} & 53,17 & 50,00 & 0,377 \\
\hline & $\begin{array}{l}45,00 \\
55,00\end{array}$ & $\begin{array}{l}66,67 \\
33,33\end{array}$ & $0,000 *$ \\
\hline $\begin{array}{l}\text { Causa externa } \\
\text { E812 } \\
\text { E815 } \\
\text { E816 }\end{array}$ & $\begin{array}{l}48,13 \\
21,66 \\
30,21\end{array}$ & $\begin{array}{l}33,51 \\
38,52 \\
27,97\end{array}$ & $0,000 *$ \\
\hline $\begin{array}{l}\text { Diseño de carretera } \\
\text { Recta ascendente } \\
\text { Recta descendente } \\
\text { Curva izq. ascendente } \\
\text { Curva izq. descendente } \\
\text { Curva der. ascendente } \\
\text { Curva der. descendente }\end{array}$ & $\begin{array}{r}7,43 \\
11,67 \\
10,08 \\
33,42 \\
7,43 \\
29,97\end{array}$ & $\begin{array}{r}9,56 \\
10,34 \\
9,56 \\
29,46 \\
12,14 \\
28,94\end{array}$ & 0,241 \\
\hline
\end{tabular}

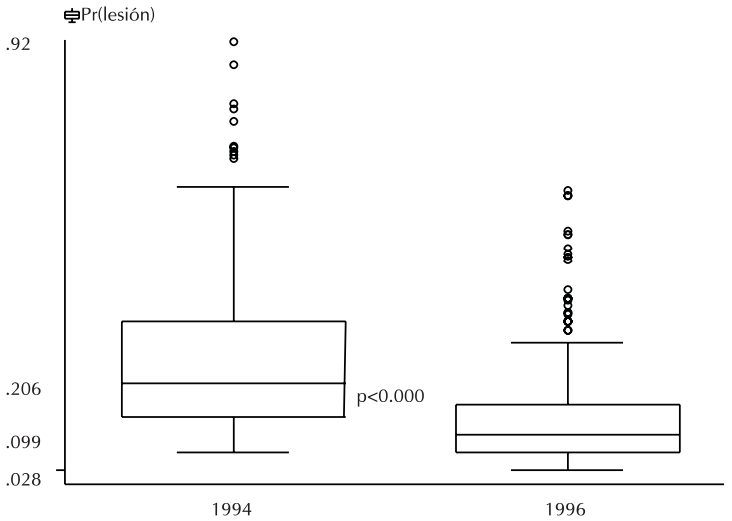

* Ajustado de acuerdo al modelo de multivariado de regresión logística.

Figura 1 - Probabilidad* de sufrir lesiones a consecuencia de un accidente de tráfico en la autopista 1994 y 1996.

\section{DISCUSIÓN}

La inclusión de los individuos al estudio estuvo condicionada a que fueran atendidos por los servicios médicos de CAPUFE, lo que pudo haber originado un sesgo de selección. Por lo anterior, los autores realizaron un ejercicio asumiendo que un $10 \%$ de los accidentes no habían sido captados por los servicios médicos, planteando 3 escenarios posibles: ningún conductor estaba lesionado; todos eran lesionados leves; y todos eran lesionados graves. Los análisis mostraron que en ninguna de las 3 situaciones, las odds ratios para los principales factores de riesgo - uso de cinturón de seguridad, ingestión de alcohol y velocidad excesiva - se alteraron en más de un $4 \%$, indicando que estas pérdidas no podrían haber afectado las conclusiones del estudio. Algunos de los sesgos de medición en el 
Tabla 3 - O dds ratios (OR) crudas de las variables significativas en la ocurrencia de lesiones por accidentes de tráfico en la autopista México-Cuernavaca, 1994 Y 1996.

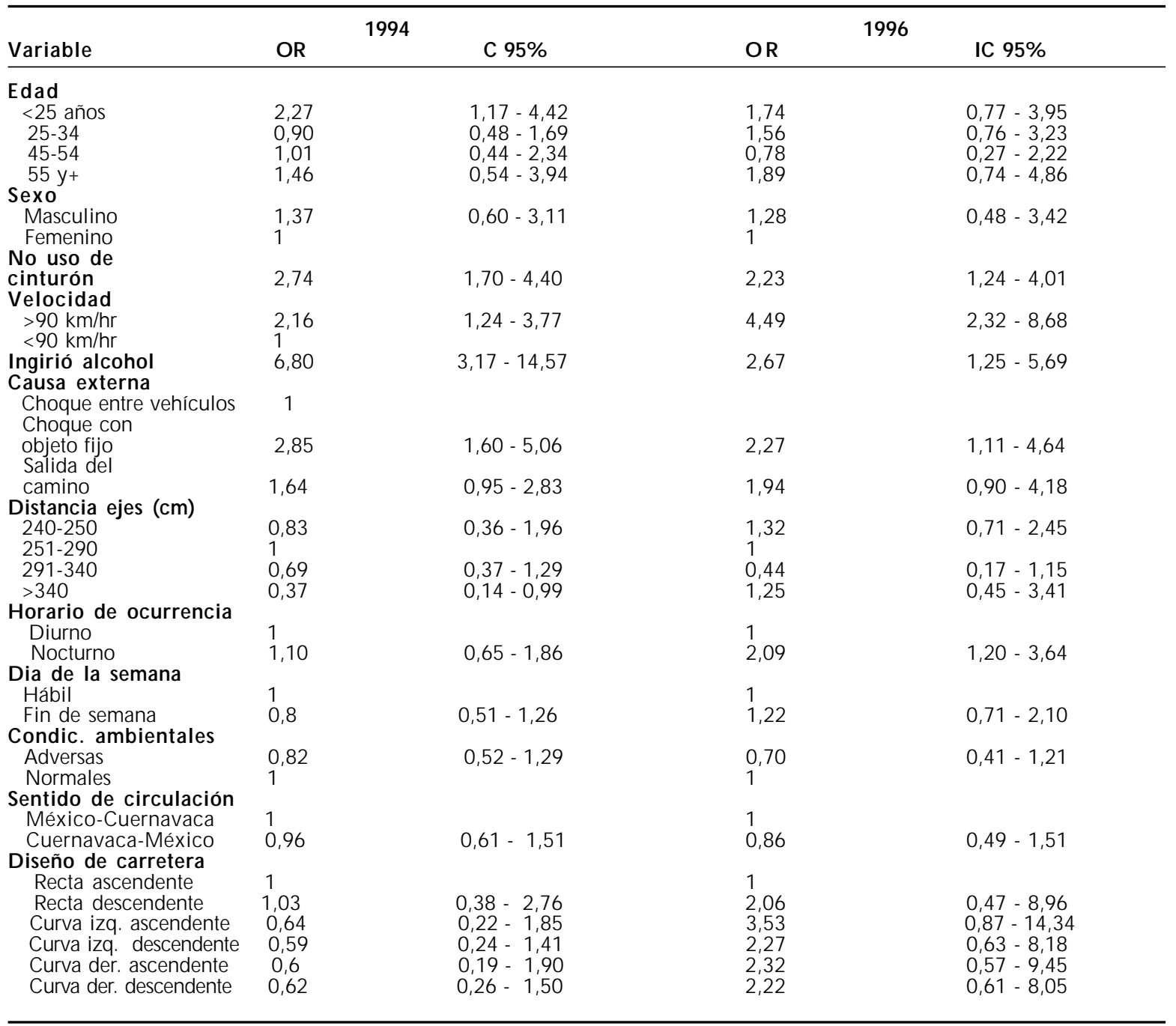

Tabla 4 - O dds ratios ajustados de lesion por accidente de tráfico en la autopista 1994-1996 - Modelo multivariado.

\begin{tabular}{|c|c|c|}
\hline Variable & OR & IC 95\% \\
\hline $\begin{array}{l}\text { Edad } \\
<25 \text { años } \\
25-34 \\
34-44 \\
45-54 \\
55 \text { y + } \\
\text { No uso de cinturón } \\
\text { Velocidad } \\
>90 \mathrm{~km} / \mathrm{hr} \\
<90 \mathrm{~km} / \mathrm{hr} \\
\text { Ingiró alcohol } \\
\text { Causa externa } \\
\text { Choque entre vehículos } \\
\text { Choque con objeto fijo } \\
\text { Salida del camino } \\
\text { Intervención } \\
\text { Log Likelihood = -283.1123 } \\
\text { goodness-of-fit test } \\
\text { Hosmer-Lemeshow chi2(4) } \\
\text { Sensibilidad } \\
\text { Especificidad } \\
\text { Valor predictivo positivo } \\
\text { Valor predictivo negativo } \\
\text { Clasifica correctamente } \\
\text { Area bajo la curva ROC }\end{array}$ & $\begin{array}{l}3,22 \\
1 \\
3,14 \\
1 \\
2,89 \\
1,76 \\
0,42 \\
33 \\
1\end{array}$ & $\begin{array}{l}1,70-4,92 \\
1,03-2,98 \\
0,27-0,66 \\
\text { Prob > chi2 =0,0000 } \\
\text { Prob > chi2 =0,6021 } \\
\text { Prob > chi2 =0,7962 } \\
\operatorname{Pr}(+\mid \mathrm{D}) 17,05 \% \\
\operatorname{Pr}(-\mid \sim \mathrm{D}) 98,16 \% \\
\operatorname{Pr}(\mathrm{D} \mid+) 68,75 \% \\
\operatorname{Pr}(\sim \mathrm{D} \mid-) 83,28 \% \\
82,59 \% \\
0,7400\end{array}$ \\
\hline
\end{tabular}

estudio ya se han discutido en un artículo previo ${ }^{9} \sin$ embargo se considera que estos tipos de sesgos no son atribuíbles a la investigación en si, sino al problema estudiado. Dichos sesgos podrán irse en la medida en que la investigación sobre el tema de los accidentes de vehículos a motor vaya avanzando.

Pocas veces es posible realizar un análisis como el que es objeto de este artículo, donde los resultados obtenidos permiten plantear posibles explicaciones a los cambios observados en la ocurrencia de lesiones provocadas por accidentes de tráfico en carretera donde se tiene documentada la realización de una intervención sobre la vía. El hecho de haber contado con variables medidas a través del mismo instrumento, con la misma metodología y aplicada por los mismos encuestadores, durante el mismo período (en meses del año), en dos momentos distintos, posibilitó contar con el antes y el después de las intervenciones 
(realizadas por el sector de Comunicaciones y Transportes), lo cual debe ser aprovechado en dos vertientes: por un lado difundir los resultados y aplicarlos en otras carreteras del país y por el otro, dejar clara la necesidad de evaluar las intervenciones que modifican el entorno físico y que tienen repercusiones en la salud de la población a través de métodos adecuados desde el punto de vista técnico y científico y que reúnan las condiciones básicas para su adecuada selección. Lo anterior también deja claro el hecho de que no es necesariamente a través del uso de diseños epidemiológicos complejos que se puede lograr posibles explicaciones a los cambios observados en un fenómeno y que la oportunidad de realizar similares mediciones tanto de la ocurrencia del accidente y su repercusión, como de los factores que los favorecen dejan claro el peso que representa una intervención, no planeada con fines de salud.

Diversos estudios han mostrado que las intervenciones para modificar los factores de riesgo del individuo como son el uso del cinturón de seguridad ${ }^{4}$, el no conducir bajo el efecto del alcohol ${ }^{6}$ o drogas, o aquellos que afecten su habilidad en la conducción del vehículo como es la experiencia o los daños físicos ${ }^{14,15}$, son por lo general a largo plazo y requieren un tiempo prolongado y con frecuentes refuerzos para mantener el efecto, y en ocasiones es a través de las generaciones que se modifican estos factores.

Por otro lado las modificaciones de los vehículos para implementar medidas de protección más eficientes lleva varios años y es determinado más por factores comerciales, que directamente por factores preventivos ${ }^{20}$. Sin embargo las modificaciones de los factores de la vía si tienen un impacto en los riesgos más inmediato, ya sea éste en sentido negativo o positivo. En este último caso la realización de acciones preventivas modificaron tanto las tasas de ocurrencia, como el riesgo de lesión por el accidente, lo que, por el tiempo entre las dos investigaciones no puede ser atribuido, a cambios importantes en los factores del individuo o del vehículo.

Los resultados permiten apoyar, al igual que en estudios realizados en otros países ${ }^{12}$, las características de la carretera, determinadas por sus condiciones de mantenimiento físico, el trazo, la señalización y las medidas de protección a la vía y a los peatones tiene un efecto no sólo sobre la ocurrencia de un accidente de tráfico, sino en el hecho de que el conductor o sus acompañantes resulten lesionados y la gravedad de las lesiones, hechos que deban ser considerados tanto en el diseño de las investigaciones como en la planificación de estrategias preventivas.

El estudio ha permitido reevaluar la participación en la gravedad de las lesiones, de los factores dependientes del conductor o de su actitud al manejar, resultando constante la asociación con el consumo de bebidas alcohólicas ${ }^{10}$ y la falta de uso del cinturón de seguridad ${ }^{8}$. La primera como consecuencia directa en la gobernabilidad del vehículo y en la capacidad de respuesta ante un trauma. La segunda como medida preventiva que disminuye el impacto de la desaceleración a la que se somete un individuo al sufrir un choque ${ }^{3}$.

La falta de asociación entre las condiciones ambientales y el resultar lesionado una vez ocurrido el accidente, demuestra que las posibles asociaciones se deberán buscar a partir de estudios posteriores entre condiciones ambientales y la ocurrencia de accidentes.

El estudio de los accidentes de tráfico se ha venido realizando desde distintas perspectivas, dependiendo del sector que lo observe, ya sea éste el de transporte, educativo, salud, y otros, todo ello producto de la multicausalidad de los mismos, lo que conduce a la realización de acciones aisladas ante el fenómeno, sin considerar que las mismas puedan tener impacto en otros sectores inclusive de tipo negativo, así, por ejemplo, el mejorar la velocidad de una vía, puede incrementar la probabilidad de la ocurrencia del accidente y/o la gravedad de las lesiones resultantes. Por ello, es evidente la necesidad de abordajes multisectoriales en el estudio de los factores causales y en la evaluación de las medidas aplicadas a su corrección, lo que permitiría tener una mayor amplitud en la medición de los efectos producidos por las acciones de los diversos sectores participantes, siendo la presente investigación un ejemplo claro de las repercusiones en salud que pueden tener las intervenciones del sector de comunicaciones en una autopista. 


\section{REFEREN CIAS}

1. Baker S, O'N eill B, Ginsburg M, Li G. The injury fact book. 2nd ed. New York, NY, Oxford U niversity Press, 1992.

2. Chías L. Las externalidades como problema emergente del sistema de transporte metropolitano. In: Castillo M, Reyes S. Problemas emergentes de la Zona Metropolitana de la Ciudad de México. UNAM, Consejo Mexicano de Ciencias Sociales A.C. Méxic0,1997. p 235-59.

3. Farmer CM, Braver ER, Mitter EL. Two-vehicle side impact crashes: the relationship of vehicle and crash characteristics to injury severity. Accid Anal Prev 1997;29:399-406.

4. Foss R, Beirness DJ, Sprattler K. Seat belt use among drinking drivers in Minnesota. Am J Public Health, 1994;84:1732-7.

5. Frenk J. Economia y Salud. Fundación Mexicana para la Salud. México,1995.

6. Glucksman E. Alcohol and accidents. Br Med Bull 1994;50:76-84.

7. Haddon W. O ptions for the prevention of motor vehicle crash injury. Isr J Med Sci 1980;16:45-65.

8. Híjar-Medina MC, Flores-Aldana ME, Lopez-Lopez MV. Cinturón de seguridad y gravedad de lesiones en accidentes de tráfico en carretera. Salud Publica M ex 1996;38:118-27.
9. Híjar-Medina MC, Lopez-Lopez MV, FloresAldana M, Anaya R. Aspectos metodológicos en la medición de los accidentes de tráfico en el sitio de ocurrencia. Rev Saude Publica 1997; 31:100-4.

10. Híjar-Medina MC, Flores-Aldana ME, Lopez-Lopez MV, Rosovsky $\mathrm{H}$. Alcohol intake and severity of injuries on highways in México: a comparative analysis. Addiction 1998;93:1543-54.

11. Robertson LS. Injury Epidemiology. Oxford U niversity Press N.Y. 1992.

12. Shankar V, Mannering F, Barfield W. Effect of roadway geometrics and environmental factors on rural freeway accident frequencies. Accid Anal Prev 1995;27:371-89.

13. Stevenson MR, Jamrozik KD, Spittle J. A case-control study of traffic risk factors and child pedestrian injury. Int J Epidemiol 1995;24:957-64.

14. Waller JA. Health status and motor vehicle crashes. N Engl J Med 1991;391:554-5.

15. Williams AF, Carten O. Driver age and crash involvement. Am J Public Health 1989;79:326-7.

16. Yunes J. Mortality from violent causes in the Americas. Bull Pan Am Org 1993;27:154-67. 